\title{
Systemic Lupus Erythematosus, 5th Edition
}

Robert G. Lahita, George Tsokos, Jill P. Buyon, and Takao Koike, editors; New York: Academic Press, November 2010, 1154 pages, \$250 US

The introduction to Lahita's Systemic Lupus Erythematosus, 5th edition, warns that only "three simple things can be said about SLE; the disease favors women, has a genetic association, and has an elusive trigger." This statement sets the tone for this authoritative tome, which adeptly balances confident, exhaustive review of what is known with deferential acknowledgment of what remains to be learned of this complex disease.

Lahita has expertly unified the breadth and depth of topics addressed with fluid and intuitive organization: the text is divided broadly into parts on SLE pathogenesis, clinical aspects, and treatment. These, in turn, are divided into chapters that are informed by the preceding sections, and inform those that appear later, but whose subheadings can be read as stand-alone summaries. This translates into impressive utility for readers, from those seeking basic appreciation of the disease to those with mastery; the text is geared to a treating rheumatologist or a basic scientist in the field of autoimmunity. Diagrams and tables are lucid and well described; photographs are abundant, not superfluous, and impressively highlight the protean visible manifestations of SLE.

There is an elegant and logical flow to the sections on pathogenesis, with chapters outlining basic immunogenetic principles, paving the way for their application through the sections pertaining to environmental aspects of pathogenesis and to mechanisms of tissue injury. The sections devoted to the clinical aspects of disease are most impressive for their commitment to addressing practical, real-life clinical queries, from monitoring to measuring outcome to thoughtful, explicit exploration of controversial areas. Outstanding chapters worthy of special mention include those addressing lupus nephritis, neuropsychiatric lupus, and pregnancy and reproductive concerns in patients with SLE. The section dedicated to the antiphospholipid syndrome is a boon to the treating clinician.

The text boasts $90 \%$ new authorship contributing new topics and rendering this reference state of the art in its weighty focus on disease pathogenesis, systems-based mechanisms of tissue injury, and treatments. While it is true that very few simple things can be said of SLE, this deftly crafted text stays true to Albert Einstein's adage that "everything should be made as simple as possible, but not simpler." Thus the fifth edition of Lahita's Systemic Lupus Erythematosus should rightfully remain a preeminent reference for its relevance, completeness, and clarity.

AMANDA J. STEIMAN, MD, FRCPC, Rheumatology Fellow, University of Toronto, Centre for Prognosis Studies in the Rheumatic Diseases, Toronto Western Hospital, Toronto, Ontario, Canada

J Rheumatol 2011;38:8; doi:10.3899/jrheum.110551 